\title{
1 HTSeqQC: A Flexible and One-Step Quality Control Software for High-throughput
}

2 Sequence Data Analysis

3

4 Renesh Bedre ${ }^{1}$, Carlos Avila², Kranthi Mandadi ${ }^{1,3^{*}}$

5

$6{ }^{1}$ Texas A\&M AgriLife Research and Extension Center, Texas A\&M University, Weslaco, TX,

7 USA

$8 \quad{ }^{2}$ Department of Horticultural Science, Texas A\&M University, College Station, TX, USA

$9{ }^{3}$ Department of Plant Pathology and Microbiology, Texas A\&M University, College Station, TX, 10 USA

11

12 Running title: Quality Control of High-throughput Sequence Data

13

14 *corresponding author: kkmandadi@tamu.edu

15

16 Keywords: High-throughput sequencing (HTS), Quality control, Parallel computing, Software

17 tool, CyVerse, Nextflow

18

19

20

21

22

23

24

25

26

27

28

29

30

31 


\section{ABSTRACT}

34 Use of high-throughput sequencing (HTS) has become indispensable in life science research.

35 Raw HTS data contains several sequencing artifacts, and as a first step it is imperative to remove

36 the artifacts for reliable downstream bioinformatics analysis. Although there are multiple stand-

37 alone tools available that can perform the various quality control steps separately, availability of

38 an integrated tool that can allow one-step, automated quality control analysis of HTS datasets

39 will significantly enhance handling large number of samples parallelly. Here, we developed

40 HTSeqQC, a stand-alone, flexible, and easy-to-use software for one-step quality control analysis

41 of raw HTS data. HTSeqQC can evaluate HTS data quality and perform filtering and trimming

42 analysis in a single run. We evaluated the performance of HTSeqQC for conducting batch

43 analysis of HTS datasets with 322 sample datasets with an average $\sim 1 \mathrm{M}$ (paired end) sequence

44 reads per sample. HTSeqQC accomplished the QC analysis in 3 hours in distributed mode and

$45 \sim 31$ hours in shared mode, thus underscoring its utility and robust performance. In addition to

46 command-line execution, we integrated HTSeqQC into the free, open-source, CyVerse

47 cyberinfrastructure resource as a GUI interface, for wider access to experimental biologists who

48 have limited computational resources and/or programming abilities. 


\section{INTRODUCTION}

64 Advancements in high throughput sequencing (HTS) technologies transformed biological research. HTS largely replaced conventional low-throughput Sanger-based sequencing technologies for genome-scale studies. Multiple genome sequencing approaches (DNA-seq,

67 RAD-seq, GBS, AgSeq) are being used to study genetic variations, discovery of novel genes, high-throughput genotyping, biomarker discovery, and precision medicine ${ }^{1-5}$. Similarly, transcriptome-level sequencing approaches (RNA-seq) allows determining the steady-state expression of genes, identification of novel transcripts and isoforms, alternatively splicing patterns, polymorphisms, gene co-expression networks, allele-specific expressions, and long non-coding RNAs (lincRNA) ${ }^{6-9}$. Illumina’s benchtop and production-scale sequencers, which are by far the most widely used HTS platforms, can generate up to 1 and 20 billion sequence reads per run, respectively. This sequencing output is expected to rapidly increase due to further advancements in the Illumina technologies. As such advanced bioinformatics tools are necessary to accelerate the analysis and efficiently manage the large volumes of data generated by the Illumina sequencing platforms.

In all HTS experiments, a critical first step is raw data quality assessment, filtering and trimming. HTS raw data often contains sequences of poor quality along with adapter or primer contaminations, and uncalled bases $(\mathrm{N})$, which if not removed can significantly hamper the downstream bioinformatics analysis leading erroneous conclusions ${ }^{10-12}$. Several standalone

82 software tools are available for quality control analysis, trimming, and filtering of HTS data such

83 as FastQC (https://www.bioinformatics.babraham.ac.uk/projects/fastqc/), NGS QC ${ }^{13}$, FASTX-

84 Toolkit (http://hannonlab.cshl.edu/fastx_toolkit/), NGS QCbox ${ }^{14}$, Trimmomatic ${ }^{15}$, fastp ${ }^{16}$, and

85 QC-Chain ${ }^{11}$. However, most of them have limitations. For instance, FastQC performs only

86 quality check of the data, and does not filter or trim of raw sequences

87 (https://www.bioinformatics.babraham.ac.uk/projects/fastqc/). Conversely, FASTX-Toolkit

88 although is equipped to perform quality filtering does not support parallel computing to handle

89 large-scale batch analysis. Other software tools such as NGS QC, QC-Chain, Trimmomatic, and

90 NGS QCbox have limited features for quality filtering, handles few samples at a time, dependent

91 on other open-source software tools, and have a need to be run separately for different quality

92 control features. Even though fastp has advantages over other tools, it does not offer support

93 handling reads with excessive uncalled bases and does not support multiplexing or batch analysis 
${ }^{16}$. With HTS becoming more accessible and affordable, it is crucial to develop a flexible and

95 integrative tool that can not only perform thorough quality control analysis, but can handle several hundred samples parallelly, with the least number of data handling steps.

Here, we present HTSeqQC, which is an open-source and easy-to-use quality control analysis software tool for cleaning raw HTS datasets generated from Illumina sequencing platforms. HTSeqQC is a flexible, one-step quality control software tool and can handle large number of samples. HTSeqQC integrates filtering and trimming modules for single and paired end HTS data and supports parallel computing for batch quality control analysis. In addition to the quality filtering and trimming analysis, HTSeqQC generates statistical summaries and visualization to assess the quality of the HTS datasets. HTSeqQC can be used as command-line interface (CLI) as well as GUI. The GUI is available through CyVerse ${ }^{17,18}$ Discovery Environment (https://cyverse.org/).

\section{RESULTS AND DISCUSSION}

\section{Case studies}

109 To evaluate the performance of HTSeqQC for quality control analysis, we analyzed several 110 datasets using a 64 GB RAM and 20 CPUs computing node. First, we analyzed a single-end raw

111 RNA-seq dataset generated on Illumina HiSeq 2000 platform corresponding to cotton, a dicot 112 plant (BioProject accession PRJNA275482 and SRA ID: SRR1805340, Table 1) ${ }^{19,20}$. HTSeqQC

113 analysis was performed with the default parameters. HTSeqQC automatically detected the

114 Illumina sequence quality variant and filtered out the reads with Phred quality score $\leq 20$. In total, HTSeqQC filtered out $~ 5 \mathrm{M}$ reads (Fig.3 and Supplementary File 1A).

Second, we evaluated multiple paired-end raw RNA-seq datasets generated on Illumina

117 HiScanSQ platform corresponding to sugarcane, a monocot plant (BioProject accession PRJNA291816 and SRA IDs: SRR2165176, SRR2165177, SRR2165178) ${ }^{6,21}$. Initially, we ran

119 HTSeqQC on one paired-end dataset (SRR2165176) using default parameters (Table 1).

120 HTSeqQC was able to analyze the Illumina sequence quality variants and filtered out the reads 121 with Phred quality score $\leq 20$ (Supplementary File 1B). For instance, the HTSeqQC filtered out 122 the $250 \mathrm{~K}$ sequence reads which were below the quality threshold (Supplementary File 1B). 123 Next, we ran the HTSeqQC with customized parameters for filtering adapter sequences, quality 124 thresholds, and uncalled bases. In total, HTSeqQC filtered out $\sim 451 \mathrm{~K}$ reads and trimmed $\sim 20 \mathrm{~K}$ 
125

126

127

128

129

130

131

132

133

134

135

136

137

138

139

140

141

142

143

144

145

146

147

148

149

150

151

152

153

154

reads (Supplementary File 1C). All the HTSeqQC commands used for performing the above analyses are provided in the README file.

In addition to quality control analysis, we also evaluated the processing time and batch handling of HTSeqQC using default parameters. HTSeqQC took $\sim 9$ min to perform the quality filtering analysis of $\sim 9 \mathrm{M}$ paired-end sequence reads, and $\sim 40$ min for $\sim 82 \mathrm{M}$ single-end sequence reads with 18 CPUs (Table 1 ).

Lastly, we analyzed 322 paired-end genotyping-by-sequencing (GBS) datasets corresponding to tomato ${ }^{5}$. These datasets had $\sim 1 \mathrm{M}(\times 2)$ sequence reads per sample. For this experiment, we also compared parallel computing in shared vs. distributed mode with 18 CPUs per computing node using Nextflow. We were able to analyze the 322 datasets in $\sim 1855 \mathrm{~min}$ ( $\sim 31$ hours) and $\sim 157 \mathrm{~min}$ ( $\sim 3$ hours) in the shared and distributed computing modes, respectively (Table 1).

\section{Advantages of HTSeqQC over existing quality control analysis software}

We compared the advantages of HTSeqQC with prevailing quality control analysis tools such as the FastQC (https://www.bioinformatics.babraham.ac.uk/projects/fastqc/), NGS QC ${ }^{13}$, QC Chain ${ }^{11}$, FASTX-Toolkit (http://hannonlab.cshl.edu/fastx_toolkit/), fastp ${ }^{16}$ and NGS QCbox ${ }^{14}$ on various important quality control features (Table 2). Many of the current tools are not as integrated as HTSeqQC (Table 2).

For instance, programs such as FastQC only provides the quality control statistics of the raw HTS data and cannot be used for quality filtering. The FASTX-Toolkit does quality filtering, but the various trimming, filtering options are not integrated, and each analysis must be performed separately. The NGS QCbox comes close, however only allows sequence read trimming based on a quality threshold for specific window size and it is highly dependent on other open-source software tools for quality control analysis ${ }^{14}$. NGS QC allows filtering and trimming of low-quality reads similar to FASTX-Toolkit, however these software tools are independent and must be run separately. The fastp provides single run quality control analysis of FASTQ files similar to HTSeqQC but does not offer key features such as multiplex/batch quality control analysis and handling of sequence reads containing uncalled bases (N). fastp does offer other features which are not included in HTSeqQC such as poly tail trimming, UMI 
preprocessing, and basic support for the long-read sequence data. Hence, in this context, the two tools can be complementary to each other for a range of applications. accuracy of the sequence reads ${ }^{22}$. HTSeqQC has this feature of filtering the sequence reads

159 based on the content of the uncalled bases (N). Although NGS QC allows this analysis, it is not integrated, and must be run separately ${ }^{13}$. In addition to overcoming several of these limitations, HTSeqQC supports common features with NGS QC and NGS QCbox such as allowing inputs in

162 the compressed GZIP file format for seamless input of large datasets (Table 2). Furthermore, 163 similar to NGS QC, HTSeqQC has an integrated function to automatically detect the FASTQ 164 quality variants (Table 2). Only HTSeqQC provides a parameter to output the cleaned FASTQ 165 data in FASTA format, avoiding using additional file format conversion tools. Lastly, in addition 166 to the command line execution, we integrated HTSeqQC as a graphic user interface that is 167 accessible freely for everyone to access through CyVerse.

\section{CONCLUSION}

170 HTSeqQC is an open-source, integrated, and easy-to-use software designed for one-step quality

171 control visualization and quality filtering of raw HTS data generated using the Illumina

172 sequencing platforms. The flexiblility to detect and remove the low-quality sequences, adapter or

173 primer contamination, uncalled bases, in a single run, greatly enhances the automation of HTS

174 data analysis projects. Because HTSeqQC can be implemented by parallel computing, it enables

175 batch handling of large number (>300) of datasets. In addition to the command line interface,

176 HTSeqQC is available as graphic user interface that is accessible freely through CyVerse. The

177 later should significantly facilitate its use among biologists without much prior bioinformatics or 178 command line computing experience.

\section{MATERIALS AND METHODS}

\section{Implementation}

HTSeqQC is a standalone open-source command-line software tool developed using

183 Python 3 for quality control analysis of HTS data generated from Illumina sequencing platforms.

184 The current HTSeqQC version (v1.0) was developed specifically for Illumina generated FASTQ 185 datasets, however, it could be utilized with other sequencing platforms given the input is FASTQ 
and supports same quality formats as Illumina. We focused on Illumina mainly because it is the most-widely utilized platform. We will continue development and subsequent versions of HTSeqQC will be released to expand its use to other platforms such as PacBio and/or Nanopore Sequencing. At its core, HTSeqQC consists of two main modules that are intended to filter and trim single and paired-end sequence datasets. Both modules were implemented using parallel computation to increase the performance of quality control analysis by allocating the input workload to the multiple CPUs. By default, there are only two CPUs, however this number can be changed as per user preferences. To some extent, HTSeqQC works similar to MapReduce where it splits the large sequence file into smaller chunks, distributes the input data to multiple CPUs, and combines the input from each process to produce a final output.

HTSeqQC checks quality issues in the raw HTS datasets and performs the quality filtering and/or trimming in a single run for removing low-quality bases, adapter contamination, and uncalled bases $(\mathrm{N})$ as per the settings of the user. The entire quality control can be completed in a single input command. By default, HTSeqQC filters out the sequence reads with Phred quality score $\leq 20$. We did not add the feature of duplicate reads removal in HTSeqQC as this feature is directly related to the gene quantification and estimation of expression ${ }^{7}$. A flowchart HTSeqQC analysis is shown in Fig. 1.

HTSeqQC can handle batch analysis of multiple sequencing datasets at a time. It accepts FASTQ file format as input and produces FASTQ or FASTA file format as output. HTSeqQC also generates summary statistics and visualization outputs for the filtered cleaned HTS datasets. All the outputs by default are saved in the same directory containing the raw RNA-seq input datasets. HTSeqQC was primarily designed to run on the Linux and Mac operating systems as command-line interface CLI (Fig. 2), however, it can also run on the Windows operating systems

212 infrastructure for large-scale data analysis developed for life science research. CyVerse can be 213 accessed through world-wide-web, and users can register for free at

214 https://user.cyverse.org/register. To use the HTSeqQC as GUI on CyVerse, it is necessary to 215 have CyVerse account. Additionally, we have also provided the docker image and Nextflow template for running the HTSeqQC. 


\section{REFERENCES}

2191 Edwards, D. \& Batley, J. Plant genome sequencing: applications for crop improvement. Plant Biotechnol. J. 8, 2-9, doi:10.1111/j.1467-7652.2009.00459.x (2010). Bolger, M. E. et al. Plant genome sequencing - applications for crop improvement. Curr. Opin. Biotechnol. 26, 31-37, doi:10.1016/j.copbio.2013.08.019 (2014).

3 Suwinski, P. et al. Advancing personalized medicine through the doi:10.3389/fgene.2019.00049 (2019).

4 Awika, H. O. et al. Developing growth-associated molecular markers via high-throughput phenotyping in spinach. Plant Genome-Us 12, doi:10.3835/plantgenome2019.03.0027 (2019). and population structure of tomato (Solanum lycopersicum) germplasm developed by Texas A\&M breeding programs. Am. J. Plant Sci. 10, 1154-1180 (2019).

6 Bedre, R. et al. Genome-wide alternative splicing landscapes modulated by biotrophic sugarcane smut pathogen. Sci. Rep. 9, 8876, doi:10.1038/s41598-019-45184-1 (2019).

7 Zhou, Q., Su, X., Jing, G., Chen, S. \& Ning, K. RNA-QC-chain: comprehensive and fast quality control for RNA-Seq data. BMC Genomics 19, 144, doi:10.1186/s12864-0184503-6 (2018).

8 Bedre, R., Irigoyen, S., Petrillo, E. \& Mandadi, K. K. New era in plant alternative splicing analysis enabled by advances in high-throughput sequencing (HTS) technologies. Front. Plant Sci. 10, doi:10.3389/fpls.2019.00740 (2019).

$9 \mathrm{Xu}, \mathrm{W}$. et al. Differential expression networks and inheritance patterns of long noncoding RNAs in castor bean seeds. Plant J. 95, 324-340, doi:10.1111/tpj.13953 (2018).

10 Conesa, A. et al. A survey of best practices for RNA-seq data analysis. Genome Biol. 17, 13, doi:10.1186/s13059-016-0881-8 (2016).

11 Zhou, Q., Su, X., Wang, A., Xu, J. \& Ning, K. QC-Chain: fast and holistic quality control method for next-generation sequencing data. PLoS One 8, e60234, doi:10.1371/journal.pone.0060234 (2013).

12 Trivedi, U. H. et al. Quality control of next-generation sequencing data without a reference. Front. Genet. 5, 111, doi:10.3389/fgene.2014.00111 (2014).

13 Patel, R. K. \& Jain, M. NGS QC Toolkit: a toolkit for quality control of next generation sequencing data. PLoS One 7, e30619, doi:10.1371/journal.pone.0030619 (2012).

14 Katta, M. A., Khan, A. W., Doddamani, D., Thudi, M. \& Varshney, R. K. NGS-QCbox and Raspberry for parallel, automated and rapid quality control analysis of large-scale next generation sequencing (Illumina) data. PLoS One 10, e0139868, doi:10.1371/journal.pone.0139868 (2015).

15 Bolger, A. M., Lohse, M. \& Usadel, B. Trimmomatic: a flexible trimmer for Illumina sequence data. Bioinformatics 30, 2114-2120, doi:10.1093/bioinformatics/btu170 (2014).

16 Chen, S., Zhou, Y., Chen, Y. \& Gu, J. fastp: an ultra-fast all-in-one FASTQ preprocessor. Bioinformatics 34, i884-i890, doi:10.1093/bioinformatics/bty560 (2018).

17 Goff, S. A. et al. The iPlant collaborative: Cyberinfrastructure for plant biology. Front Plant Sci 2, doi:10.3389/fpls.2011.00034 (2011). 
26118 Merchant, N. et al. The iPlant Collaborative: Cyberinfrastructure for Enabling Data to

262 Discovery for the Life Sciences. PLoS Biol. 14, e1002342,

263 doi:10.1371/journal.pbio.1002342 (2016).

26419 Bedre, R. et al. Genome-wide transcriptome analysis of cotton (Gossypium hirsutum L.) identifies candidate gene signatures in response to aflatoxin producing fungus Aspergillus flavus. PLoS One 10, e0138025, doi:10.1371/journal.pone.0138025 (2015).

20 Bedre, R. Genome-wide transcriptome analysis of cotton (Gossypium hirsutum L.) to identify genes in response to Aspergillus flavus infection, and development of RNA-Seq data analysis pipeline Ph.D. thesis, Louisiana State University, (2016).

\section{Acknowledgements}

278 We thank Upendra Devisetty, Reetu Tuteja, and Sarah Roberts for their assistance with installing

279 HTSeqQC at CyVerse DE, which was made possible through CyVerse's External Collaborative

280 Partnership program. We acknowledge support of Texas A\&M High Performance Research

281 Computing Center (http://hprc.tamu.edu/) resources and sequencing support of the Texas A\&M

282 AgriLife Genomics and Bioinformatics Service (https://txgen.tamu.edu/). We also acknowledge

283 support of Louisiana State University Agricultural Center and Louisiana State University High

284 Performance Computing resources (http://www.hpc.lsu.edu/) for supporting early stages of the

285 HTSeqQC development as part of Ph.D. research of RB. This work was supported in part by

286 funds from Texas A\&M AgriLife Research Insect-vectored Disease Seed Grant (114190-96210)

287 to KM, Foundation for Food and Agricultural Research New Innovator Award (2018-534299)

288 and USDA-NIFA (2018-70016-28198, HATCH 1023984) awards to KM.

\section{Author contributions}

291 RB developed the software, platform and conducted the analysis. CA, and KM supervised the

292 study, data analysis and interpretation. All authors have read, reviewed, and approved the

293 manuscript.

294

\section{Competing interests}

296 All authors declare no competing interests. 


\section{Availability}

298 HTSeqQC software, Docker image and Nextflow template are available for download at

299 https://github.com/reneshbedre/HTSeqQC and graphical user interface (GUI) is available at

300 CyVerse Discovery Environment (DE) (https://cyverse.org/ ). Documentation available at

301 https://reneshbedre.github.io/blog/htseqqc.html and https://cyverse-htseqqc-cyverse-

302 tutorial.readthedocs-hosted.com/en/latest/. The HTSeqQC is also available on Anaconda cloud

303 (https://anaconda.org/bioconda/htseqqc) and can be installed using biconda channel.

305 Supplementary data

306 Supplementary information provided in Supplementary File 1.

308 Figure legends

309 Fig. 1: Flowchart of HTSeqQC analysis. HTSeqQC includes two main modules for quality

310 control analysis of single and paired-end HTS datasets generated from Illumina sequencing

311 platforms. HTSeqQC filters and trims the raw HTS datasets to remove low-quality bases, adapter

312 or primer contamination, and uncalled bases to generate high-quality datasets for downstream

313 bioinformatics analysis.

Fig. 2: Command-line interface (CLI) and Graphic-user interface (GUI) of HTSeqQC.

316 HTSeqQC can be launched as CLI or GUI modules. For CLI, the HTSeqQC need to installed on

317 a local system, whereas GUI is preinstalled and ready to use online at CyVerse

318 (https://cyverse.org/).

320 Fig. 3: The sequence quality evalaution of HTS datatsets performed by HTSeqQC. A) The

321 sequence quality distribution among the raw (unfiltered) and cleaned (filtered) sequence data,

322 B) The distribution of percenatges of sequence reads with quality score, C) The percentage GC

323 content distribution among the raw and cleaned sequence data, D) The content of nucleotide

324 bases in raw and cleaned sequence data. 
Table 1: Summary of the quality control analysis of single and paired-end datasets with single and multiple samples performed using HTSeqQC CLI.

\begin{tabular}{|c|c|c|c|c|c|}
\hline $\begin{array}{l}\text { SRA Accession or } \\
\text { Samples }\end{array}$ & $\begin{array}{l}\text { Read Type (\# } \\
\text { Samples) }\end{array}$ & $\begin{array}{l}\text { \# Sequence reads } \\
\text { (File size in } \mathrm{GB}^{\mathrm{a}} \text { ) }\end{array}$ & $\begin{array}{l}\text { Read } \\
\text { length } \\
\text { (bp) }\end{array}$ & $\begin{array}{l}\text { \# CPUs } \\
\text { (Parallel } \\
\text { computing) }\end{array}$ & Run time (Min) \\
\hline SRR2165176 & Paired (1) & $8,583,424 \times 2(5.6)$ & 100 & 18 (Shared) & 9 \\
\hline $\begin{array}{l}\text { SRR2165176 } \\
\text { SRR2165177 } \\
\text { SRR2165178 }\end{array}$ & Paired (3) & $\begin{array}{l}8,583,424 \times 2(5.6) \\
9,282,222 \times 2(6) \\
9,918,081 \times 2(6.4)\end{array}$ & $\begin{array}{l}100 \\
100 \\
100\end{array}$ & 18 (Shared) & 28 \\
\hline SRR1805340 & Single (1) & $82,059,811(27)$ & 100 & 18 (Shared) & 40 \\
\hline Tomato GBS ${ }^{b}$ & Paired (322) & $\begin{array}{l}\sim 1,000,000 \times 2 \\
(206)\end{array}$ & 150 & $\begin{array}{l}18 \text { (Shared) } \\
18 \text { (Distributed) }\end{array}$ & $\begin{array}{l}1855 \\
157\end{array}$ \\
\hline
\end{tabular}

${ }^{\mathrm{a} C o m b i n e d ~ f i l e ~ s i z e ~ o f ~ t w o ~ f i l e s ~ f o r ~ p a i r e d-e n d ~ s e q u e n c e ~ r e a d s ~}$

b322 tomato genotypes were sequenced using low-coverage whole genome sequencing by Illumina HiSeq $4000{ }^{5}$.

The number of sequences reads are average of all the datasets. 
1 Table 2: The key sequence quality features comparison of HTSeqQC with other leading and

2 equivalent quality control software tools.

\begin{tabular}{|c|c|c|c|c|c|c|c|}
\hline Features & HTSeqQC & FastQC $^{a}$ & NGS QC & QC-Chain & $\begin{array}{l}\text { FASTX- } \\
\text { Toolkit }\end{array}$ & $\begin{array}{l}\text { NGS } \\
\text { QCbox }\end{array}$ & fastp \\
\hline Low quality filtering & Yes & Yes & Yes & Yes & Yes & No & Yes \\
\hline Uncalled bases (N) & Yes & No & Yes* & No & No & No & No \\
\hline $\begin{array}{l}\text { Adapter or primer } \\
\text { trimming }\end{array}$ & Yes & Yes & Yes & Yes & Yes & No & Yes \\
\hline Multiple adapter support & Yes & Yes & Yes & Yes & No & No & Yes \\
\hline $\begin{array}{l}\text { Minimum read length } \\
\text { filtering }\end{array}$ & Yes & No & No & No & No & Yes & Yes \\
\hline Low quality trimming & Yes & No & Yes* & Yes & No & Yes & Yes \\
\hline Paired-end support & Yes & No & Yes & Yes & No & Yes & Yes \\
\hline $\begin{array}{l}\text { Paired-end sequence } \\
\text { order }\end{array}$ & Yes & No & Yes & Yes & No & Yes & Yes \\
\hline Visualization & Yes & Yes & Yes & Yes & Yes & Yes & \\
\hline Parallel computing & Yes & Yes & Yes & Yes & No & Yes & Yes \\
\hline $\begin{array}{l}\text { Automatic } \\
\text { quality variant detection }\end{array}$ & Yes & No & Yes & No & No & No & No \\
\hline FASTA Output & Yes & No & No & No & No & No & No \\
\hline Programming & Python 3 & Java & PERL & $\mathrm{C}++$ & $\mathrm{C}$ & $\mathrm{C}$ & $\mathrm{C} / \mathrm{C}++$ \\
\hline GZIP FASTQ input & Yes & No & Yes & No & No & Yes & Yes \\
\hline $\begin{array}{l}\text { Multiple sample support } \\
\text { (batch analysis) }\end{array}$ & Yes & Yes & Yes & No & No & Yes & No \\
\hline GitHub & Yes & Yes & No & No & Yes & Yes & Yes \\
\hline
\end{tabular}

$4 \quad{ }^{a}$ FastQC only provides quality metric and does not filter or trim HTS data

$5 \quad *$ Separate tools for given features 


\section{Raw High-throughput sequencing (HTS) data}

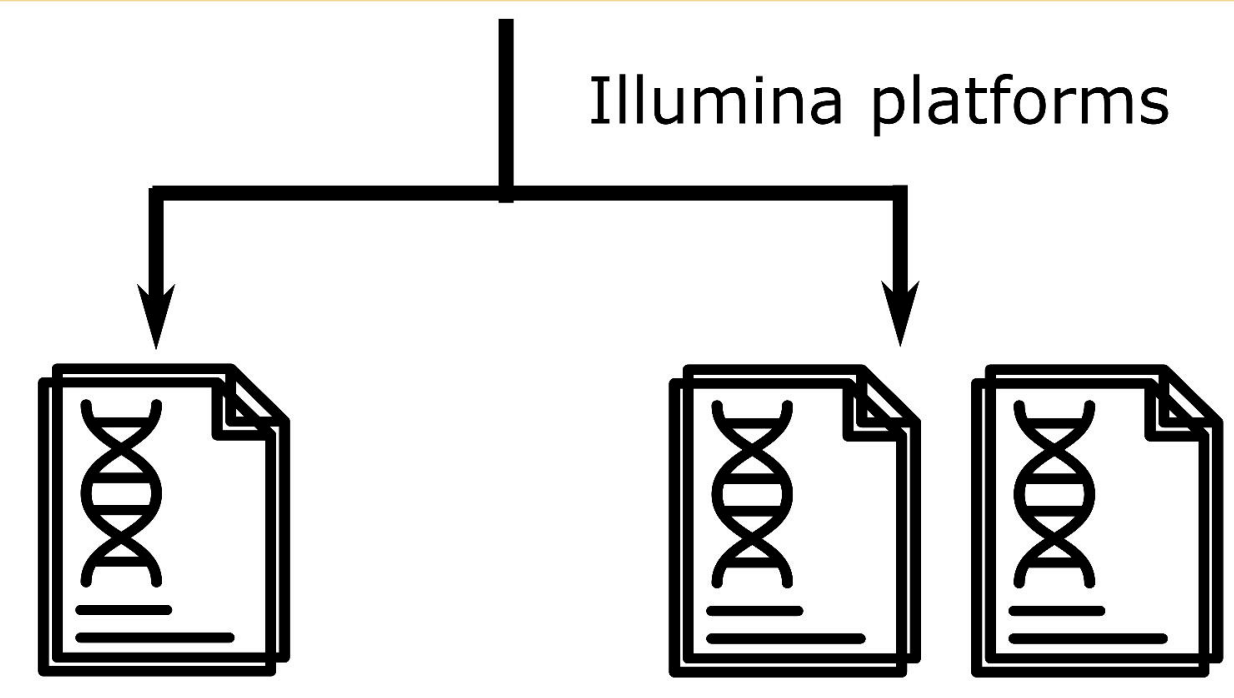

Single end

Paired end

HTSeqQC

Sequence quality trimming and filtering
CLI, GUI, Docker, and Nextflow

Parallel computing (Shared and distributed)

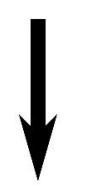

High-quality HTS data 
A $11 \mathrm{e} 7$

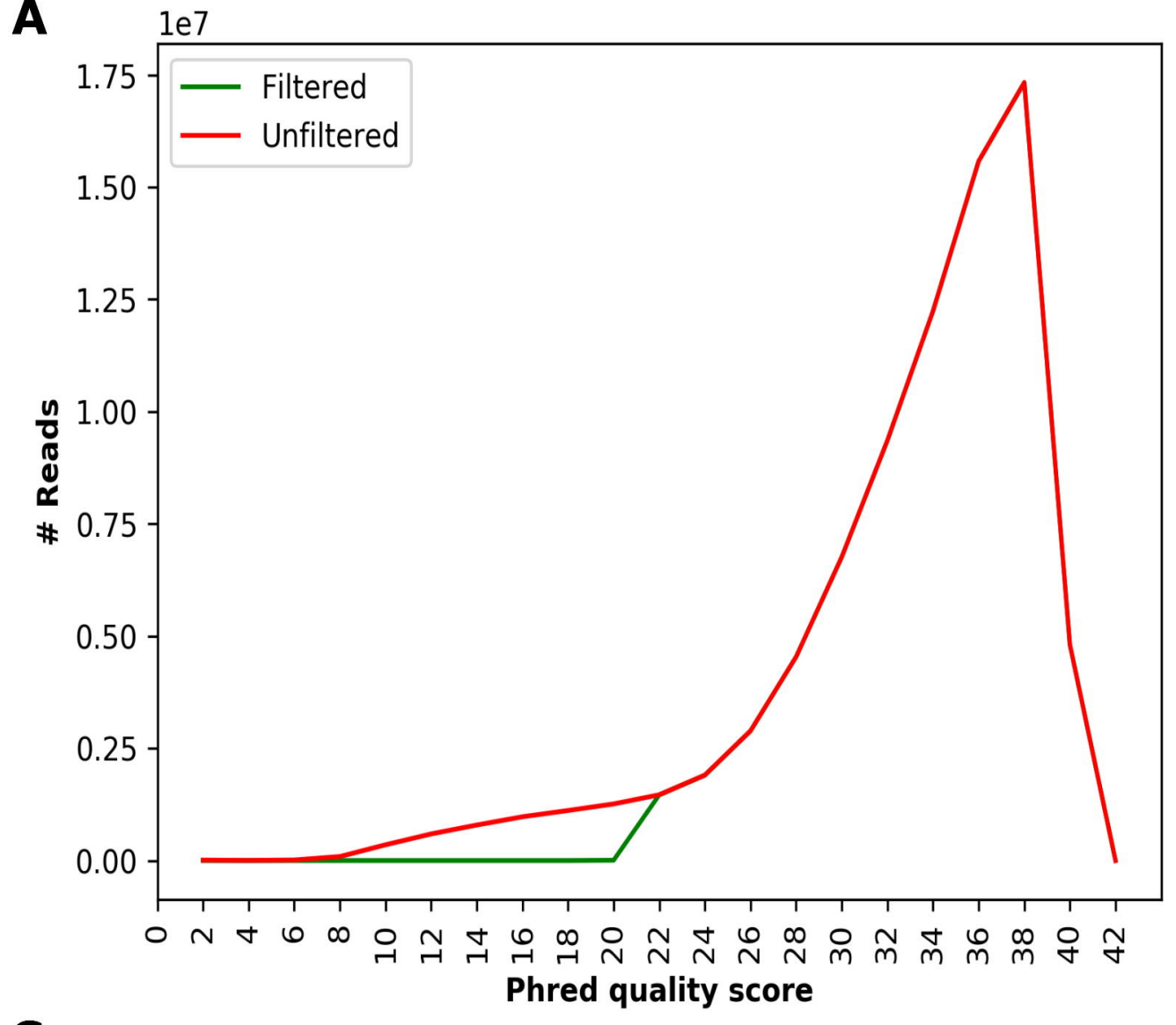

C

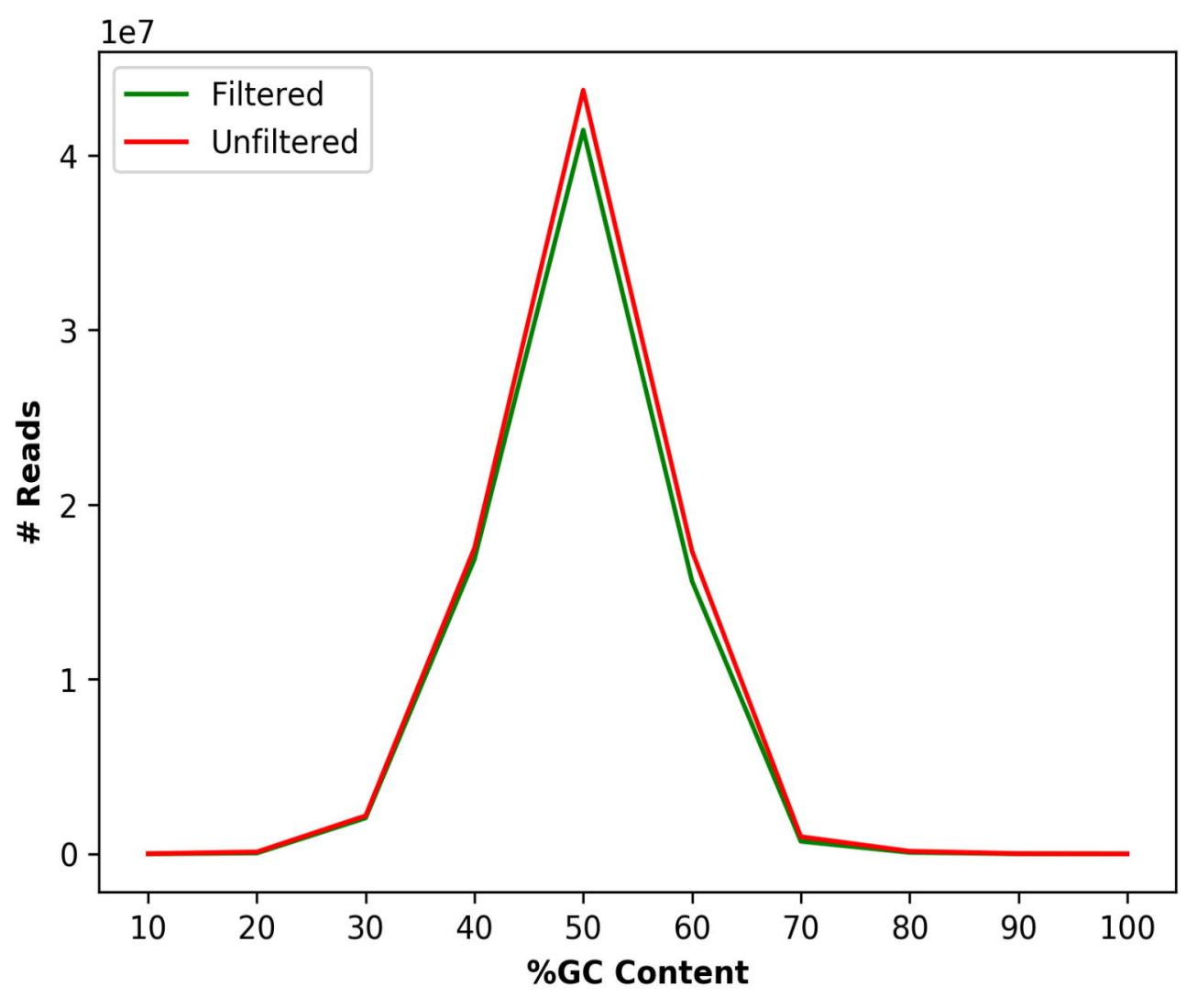

\section{Quality Score Distribution}

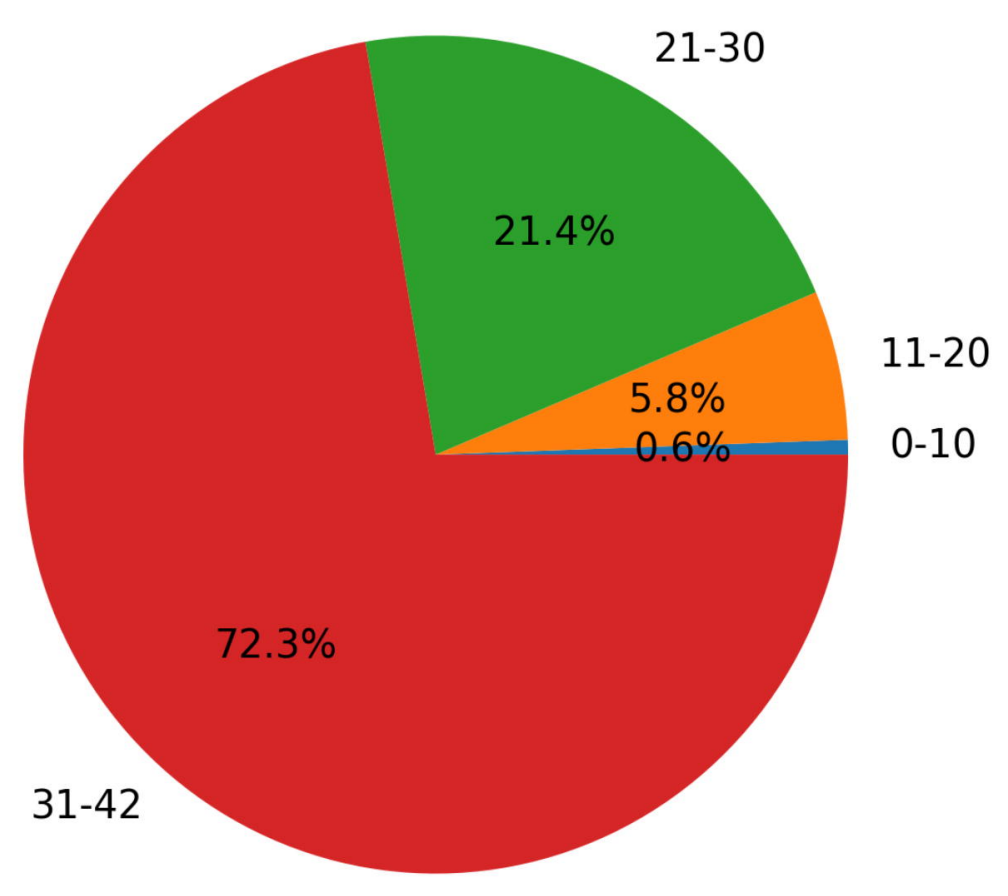

D

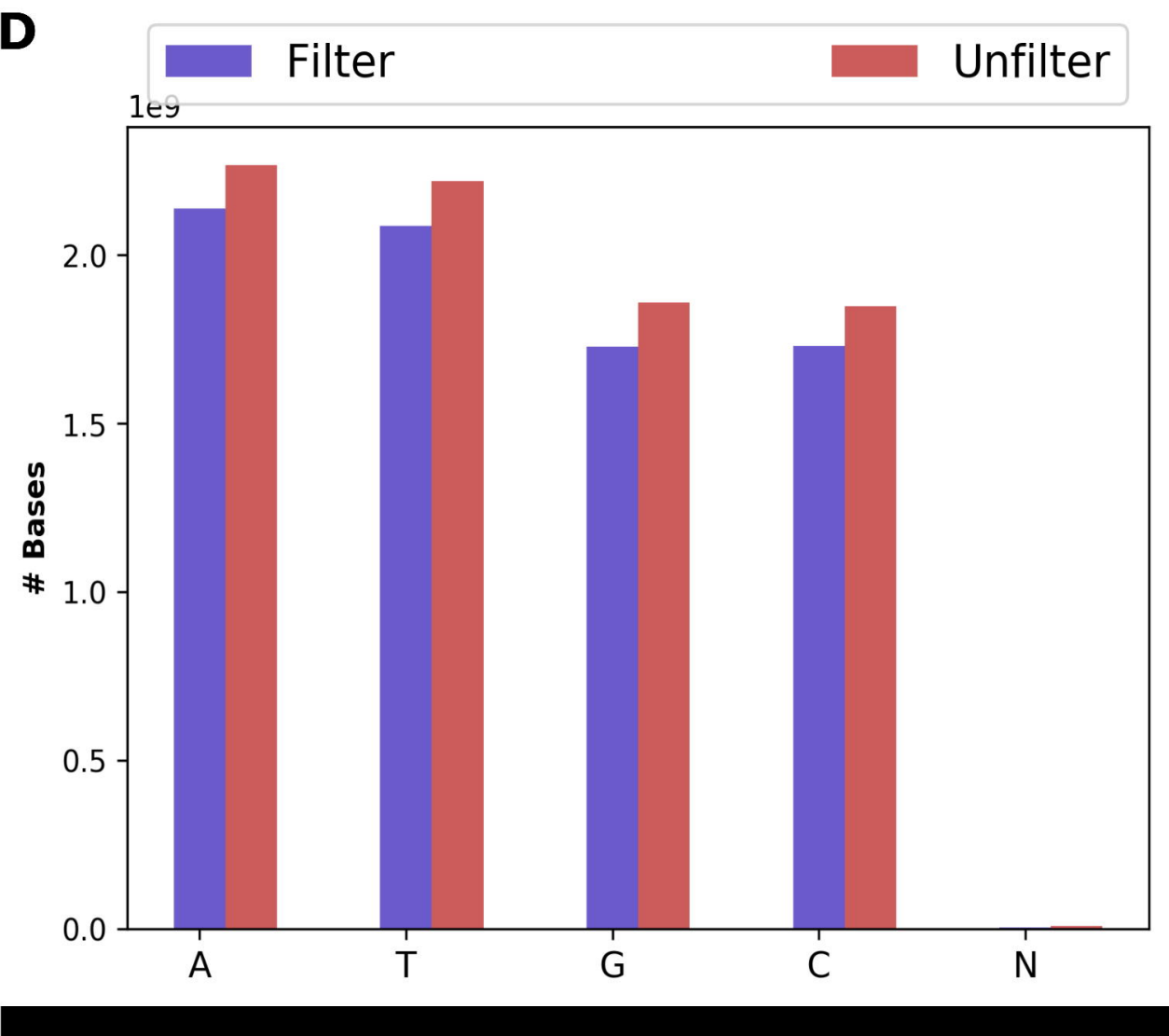

